\title{
Critical Length of a Column in View of Bifurcation Theory
}

\author{
M. Kopáčková \\ The paper investigates nonlinear eigenvalue problem for a vertical homogeneous rod loaded with its own weight only. The critical length of \\ the rod, for which the rod loses its stability, is found by use of bifurcation theory. Dependence of maximal deflections of the rods on their \\ lengths is given.
}

Keywords: critical length of column, eigenvalue problem, bifurcation theory, approximation of solution, Bessel function.

\section{Introduction}

Linear formulation of the eigenvalue problem for a vertical rod loaded with its own weight has apparent drawbacks, e.g.,

a) all the critical lengths of the rod (i.e., the eigenvalues) compose a discrete set, outside of which all the lengths are noncritical, i.e., nonzero deflections are impossible, and

b) all the critical deflections (i.e., the eigenfunctions) are of an arbitrary magnitude.

To a certain extent, they may be eliminated using the precise (nonlinear) form of the curvature. The nonlinear problem was solved long ago by L. Euler (1744) and by J. L. Lagrange (1773), who gave the solution of the simplest problem for a homogeneous rod loaded with a longitudinal force (neglecting its own weight) in the form of elliptic functions. Detailed analysis of the problem was published e.g., in [1], [2] and it is mentioned in the first part of the paper as an introduction to the bifurcation problem.

The second part of this paper contains the formulation of nonlinear eigenvalue problem for a vertical homogeneous rod loaded with its own weight only, and the corresponding linear problem is solved. The last part is devoted to applications of bifurcation theory (see e.g., [3], [4]) to the nonlinear problem and the approximate solution of the problem is found.

All the computations and pictures were performed with use of the Maple V software.

\section{Critical load of a column in view of nonlinear theory}

The problem of finding the critical vertical load, which is applied to the free end of a vertical homogeneous rod of uniform cross section fixed at the bottom is a well-known eigenvalue problem that has been explicitly solved in many mechanics textbooks. The example is mentioned here to illustrate the differences between the results of linear and nonlinear theories.

If we denote the horizontal deflection of the column by $w$ (only such displacements are taken into account), its curvature by $\rho$, the variable length measured from the free end by $s$, the length of the whole rod by $l$, the angle between the $x$-axis and the tangent line at the point $s$ of the rod by $\theta(s)$, the equation of the moments is $E I / \rho=P(w(s)-w(0))$, where $E$ is
Young's modulus, $I$ is the moment of inertia and $P$ is the above mentioned load (in the direction $x$ of the column). Differentiating (by $s$ ) the equation and substituting the relations

$$
\frac{1}{\rho}=\frac{\mathrm{d} \theta}{\mathrm{d} s}, \quad \frac{\mathrm{d} w(s)}{\mathrm{d} s}=-\sin \theta,
$$

into it, we get

$$
\frac{\mathrm{d}^{2} \theta}{\mathrm{d} s^{2}}+p^{2} \sin \theta=0
$$

where $p^{2}=P / E I$. In the case of small deflections, the equation may be approximated by the linear equation

$$
\frac{\mathrm{d}^{2} \theta}{\mathrm{d} s^{2}}+p^{2} \theta=0
$$

having (together with the appropriate boundary conditions) the discrete set of eigenvalues

$$
p_{k}^{2}=\left(\frac{(2 k-1) \pi}{2 l}\right)^{2}, \quad k=1,2, \ldots
$$

and corresponding eigenfunctions with an arbitrary magnitude. But these results do not correspond to reality (see the introduction). For this reason many engineers and mathematicians have tried to solve the nonlinear equation (2).

Using the identity

$$
\frac{\mathrm{d}^{2} \theta}{\mathrm{d} s^{2}}=\frac{\mathrm{d}}{\mathrm{d} s}\left(\frac{1}{\rho}\right)=\frac{\mathrm{d}}{\mathrm{d} \theta}\left(\frac{1}{\rho}\right) \frac{\mathrm{d} \theta}{\mathrm{d} s}=\frac{\mathrm{d}}{\mathrm{d} \theta}\left(\frac{1}{2 \rho^{2}}\right)
$$

on the left hand side of (2), integrating by $\theta$ and taking into account the boundary condition $\theta(0)=\theta_{0}, 1 / \rho\left(\theta_{0}\right)=0$, we get

$$
\frac{1}{2 \rho(\theta)^{2}}=p^{2}\left(\cos \theta-\cos \theta_{0}\right) .
$$

Computing

$$
\rho=\frac{\mathrm{d} s}{\mathrm{~d} \theta}
$$

from (3), we must choose the sign in front of the root. As the most important eigenvalue is the smallest one, we choose the minus sign on the whole interval $\left(0, \theta_{0}\right)$, and then we will consider the smallest critical force and its right neighborhood. Integrating the root of (3) and taking into account the boundary condition $s(0)=l$, we get the implicit form of the function $\theta=\theta(s)$ : 


$$
l-s(\theta)=\frac{1}{p \sqrt{2}} \int_{0}^{\theta} \frac{d \theta}{\sqrt{\cos \theta-\cos \theta_{0}}} .
$$

The value $\theta_{0}$ is obtained by use of the substitution

$$
\sin \frac{\theta}{2}=\sin \Phi \sin \frac{\theta_{0}}{2}
$$

in (4) and limiting the result for $\theta \rightarrow \theta_{0}, s \rightarrow 0$ :

$$
l p=\int_{0}^{\frac{\pi}{2}} \frac{\mathrm{d} \Phi}{\sqrt{1-\left(\sin \frac{\theta_{0}}{2} \sin \Phi\right)^{2}}}
$$

which is the implicit form of the function $\theta_{0}(p)$. It is seen, that the smallest $p$ satisfying (5) is the smallest eigenvalue $p_{1}$ of the linear problem with corresponding $\theta_{0}=0$. The dependence of the maximum deflections $w_{0} \equiv w(0)$ on the force $P$, resp. $p$ is given by the formula

$$
w_{0}=\frac{2}{p} \sin \frac{\theta_{0}(p)}{2},
$$

which is obtained by integrating the second equality of (1) with the use of (3) and the fact

$$
\frac{\mathrm{d} w}{\mathrm{~d} s}=\frac{\mathrm{d} w}{\mathrm{~d} \theta} \cdot \frac{\mathrm{d} \theta}{\mathrm{d} s}=\frac{\mathrm{d} w}{\mathrm{~d} \theta} \cdot \frac{1}{\rho}
$$

and limiting the result for $\theta \rightarrow \theta_{0}, s \rightarrow 0$ (see the Fig. 1).

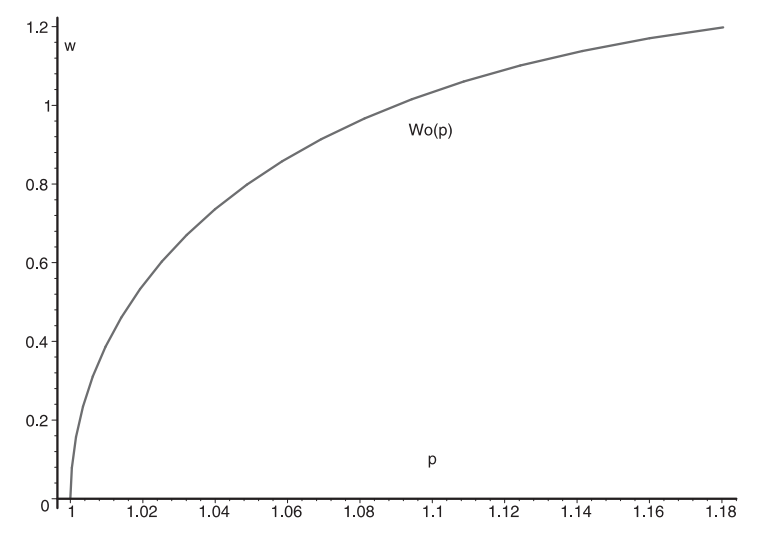

Fig. 1: Dependence of the maximum deflections $w$ on the force $P(l=\pi / 2)$

The dependence of the ratio

$$
r(p) \equiv \frac{l-x(l)}{l}
$$

on $p$ is given by the following formula

$r(p)=1-\frac{1}{l p} \int_{0}^{\pi / 2}\left(2 \sqrt{1-\left(\sin \frac{\theta_{0}}{2} \sin \Phi\right)^{2}}-\frac{1}{\sqrt{1-\left(\sin \frac{\theta_{0}}{2} \sin \Phi\right)^{2}}}\right) \mathrm{d} \theta$,

which is calculated (similarly as $w_{0}$ ) integrating the equality

$$
\frac{\mathrm{d} x(s)}{\mathrm{d} s}=\cos \theta,
$$

and limiting the result for $\theta \rightarrow \theta_{0}, s \rightarrow 0$ (see Fig. 2).

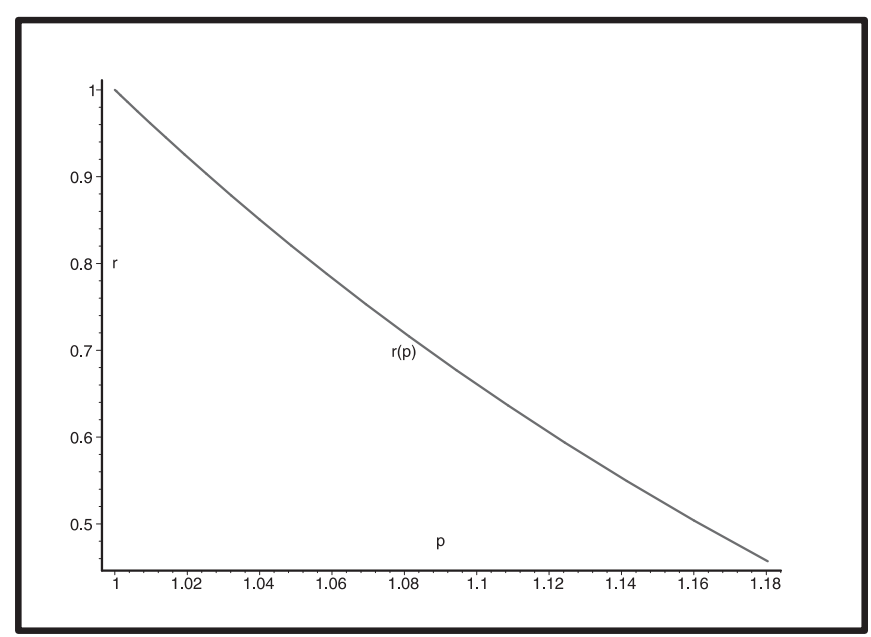

Fig. 2: Dependence of the relative extension $r$ on the force $P(l=\pi / 2)$

Two improvements comparing with the linear theory are evident:

1. If $p$ tends to the first eigenvalue $p_{1}=\mathrm{p} / 2 l$ of the linear problem from the right hand side of the interval, the maximum deflection $w_{0}$ tends to zero, whereas no deflection of the rod exists for $p \leq p_{1}$.

2. For every $p \geq p_{1}$ there exists the unique solution $w(\theta)$, $\theta \in\left(0, \theta_{0}\right]$ with maximum deflection $w_{0}(p)$.

The same considerations hold for $p_{k}, k=2,3, \ldots$

\section{Critical lengths of a column bent with its own weight - nonlinear formulation and some results of linear theory}

In the further discussion we answer the question which length of a homogeneous column of a constant cross section is bent with its own weight only, i.e., finding the smallest $l$, for which there exists nonzero deflection $w$ of the column.

Let us consider the equality of the moments in the form

$$
\frac{E I}{\rho}=\int_{0}^{s} f S(w(s)-w(\sigma)) \mathrm{d} \sigma, \quad s \in(0, l),
$$

where $f$ is the density of the column and $S$ is its cross section (the other notation coincides with that of part 1). Differentiating the equation and substituting the relations

$$
\frac{1}{\rho}=\frac{\mathrm{d} \theta}{\mathrm{d} s}, \quad \frac{\mathrm{d} w}{\mathrm{~d} s}=-\sin \theta(s)
$$

into it, we get

$$
\frac{\mathrm{d}^{2} \theta}{\mathrm{d} s^{2}}+\frac{f S}{E I} s \sin \theta(s)=0 .
$$

To eliminate the unknown length $l$ of the column, we transform $s$ to the new variable $s / l$ (denoting it again $s$ ) in the last equation and we denote the unknown function $\theta$ in the new variable by $u(s)$. Thus, we get the equation

$$
\frac{\mathrm{d}^{2} u}{\mathrm{~d} s^{2}}(s)+\lambda s \sin u(s)=0, \quad s \in(0,1),
$$


where

$$
\lambda=\frac{f S l^{3}}{E I}
$$

is an unknown eigenvalue of the problem to Equation (6) and to the boundary conditions

$$
u^{\prime}(0)=0, \quad u(1)=0 .
$$

The first condition follows from the assumption that upper end of the column is free of tension, i.e., $1 / \rho=0$, and the second condition expresses the fixed end at the bottom.

In the case of small deflections, Equation (6) may be linearized using the following approximations

$$
\sin \theta \doteq \theta \doteq w^{\prime}(s) \text {. }
$$

Thus, we get the linear eigenvalue problem

$$
\frac{\mathrm{d}^{2} v}{\mathrm{~d} s^{2}}(s)+\lambda s v(s)=0, s \in(0,1), \frac{\mathrm{d} v}{\mathrm{~d} s}(0)=0, v(1)=0,
$$

here we denoted $v(s)=w^{\prime}(s)$.

The solutions of (9) are the discrete set of the eigenvalues

$$
\lambda_{n}=\frac{9 z_{n}^{2}}{4}, \quad n=0,1, \ldots
$$

and the corresponding eigenfunctions

$$
v_{n}(s)=C \sqrt{s} J_{\dashv / 3}\left(z_{n} s^{3 / 2}\right), \quad n=0,1, \ldots
$$

where $z_{n}(n=0,1, \ldots)$ are all the roots of the Bessel function $J_{-1 / 3}(z)$ and $C$ is an arbitrary constant.

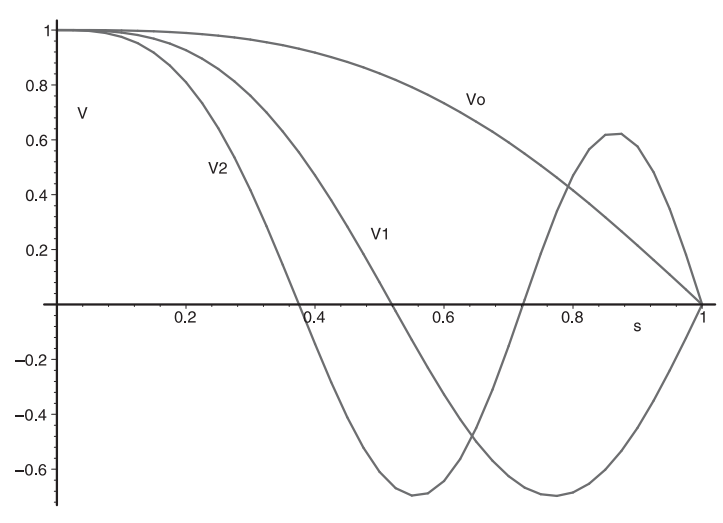

Fig. 3: Three eigenfunctions $v_{0}, v_{1}, v_{2}$ of the linear program

The first three eigenfunctions $v_{0}, v_{1}, v_{2}$ of the problem (where $C$ is chosen in such a way that $v_{i}(0)=1, i=0,1,2$ ) are given in Fig. 3, whereas the corresponding deflections $w_{0}, w_{1}, w_{2}$ are drawn in Fig. 4. The critical lengths of the column are determined by the eigenvalues $\lambda_{n}$ and the relation (7), i.e.,

$$
l_{n}^{3}=\frac{9 E I z_{n}^{2}}{4 f S} .
$$

The length for which the homogeneous column loses its stability (i.e., nonzero deflection of the column exists without any force apart from its own weight),

$$
l_{0}=\left(\frac{9 E I z_{0}^{2}}{4 f S}\right)^{1 / 3}
$$

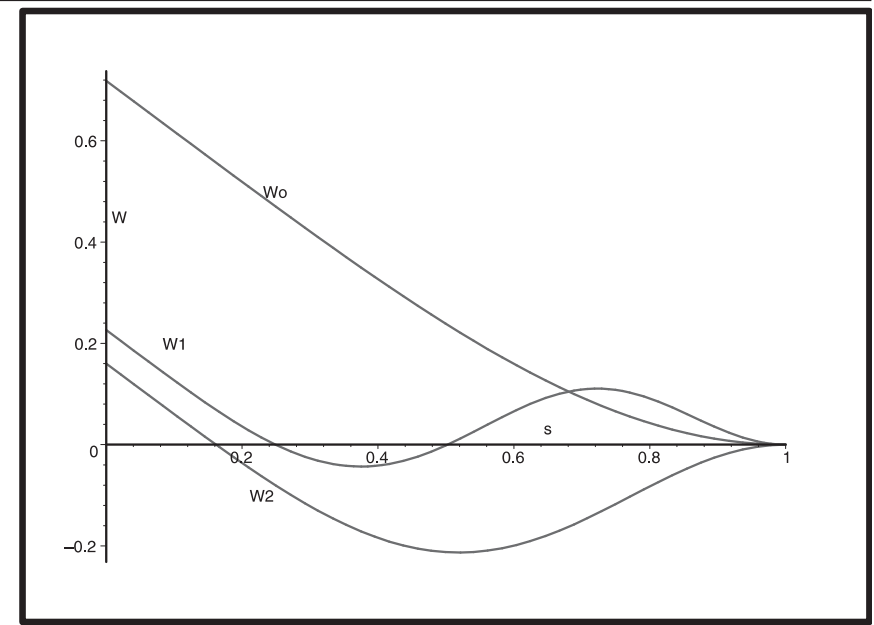

Fig. 4: Three deflections of the column

corresponds to the smallest eigenvalue $\lambda_{0}$ of the above problem, where

$$
\lambda_{0}=\frac{9 z_{0}^{2}}{4}=7.83734744
$$

and the corresponding eigenfunction is of the form

$$
v_{0}(s)=\sqrt{s} J_{\dashv / 3}\left(1.86635086 s^{3 / 2}\right), \quad s \in(0,1) .
$$

\section{The solution of the bifurcation problem}

The assumption of small deflections, and then the validity of linear theory, implies the same insufficiency as that mentioned in the introduction. To remove it, we have to take into account the nonlinear equation (6) representing together with the boundary conditions (8) the problem of a bifurcation of the nonlinear operator $L(u)-F(u, t)$ (given below) at the point $[0,0]$, (where $L(0)-F(0, t)=0$ ), i.e., there exists a nonzero solution of the equation (14) (see below) in every neighborhood of $[0,0]$.

Every eigenvalue $\lambda_{n}$ gives a bifurcation point $\left[0, \lambda_{n}\right]$ and the solution of the problem is similar to each other for $n=0,1, \ldots$ Then, we choose the smallest eigenvalue $\lambda_{0}$, because this number determines the loss of stability of the column.

Equation (1) may be rewritten into the form

$$
u^{\prime \prime}(s)+\lambda_{0} s u(s)=s\left(\lambda_{0} u(s)-\lambda \sin u(s)\right), \quad s \in(0,1) .
$$

Let us denote by $L(u)$ the linear operator as the closure (in $H^{1}(0,1)$ ) of the operator given by the values

$$
L u(s) \equiv u^{\prime \prime}(s)+\lambda_{0} s u(s)
$$

for the functions satisfying boundary conditions (8) and

$$
F(u, t)=s\left(\lambda_{0}(u(s)-\sin u(s))-\sin u(s)\right),
$$

where $=\lambda-\lambda_{0}$. Now, we may reduce the problem of finding nonzero solutions to (6), (8) for $\lambda$ near to $\lambda_{0}$ to solving the equation

$$
L u=F(u,)
$$

(in $\left.L^{2}(0,1)\right)$ for sufficiently small .

\section{All the solutions of the homogeneous equation}

$$
L u=0
$$

form a one-dimensional subspace $N$ of the eigenfunctions $v_{0}(s)$ given by the formula (11) corresponding to the eigenvalue $\lambda_{0}$. Any solution $u(s)$ to the equation 


$$
\operatorname{Lu}(s)=f(s), \quad s \in(0,1)
$$

exists and is of the form

$u(s)=b \int_{0}^{s} f(\sigma)\left(v_{0}(\sigma) u_{0}(s)-v_{0}(s) u_{0}(\sigma)\right) \mathrm{d} \sigma+C v_{0}(s)$

if and only if the function $f$ is orthogonal to $v_{0}$ in $L^{2}(0,1)$, i.e.,

$$
\int_{0}^{1} f(s) v_{0}(s) \mathrm{d} s=0,
$$

where $C$ is an arbitrary constant,

$$
b=\frac{2 \pi}{3 \sqrt{3}}, \quad u_{0}(s)=\sqrt{s} J_{1 / 3}\left(z_{0} s^{3 / 2}\right) .
$$

$u_{0}$ is a solution of

$$
u^{\prime \prime}(s)+\lambda_{0} s u(s)=0,
$$

representing together with $v_{0}$ a fundamental system of solutions to the above equation. Formula (16) is easily obtained by solving the linear problem (15), (8) with the use of variation of parameters. As operator $L$ is not invertible, we split equation (14) into two equations:

$$
\begin{aligned}
& Q(L u)=Q(F(u, t)), \\
& (I-Q) \cdot(L u-F(u, t))=0,
\end{aligned}
$$

where $Q$ is a projector operator of $L^{2}(0,1)$ on

$$
N^{\perp}=\left\{f \in L^{2}(0,1): \int_{0}^{1} f(s) v_{0}(s) \mathrm{d} s=0\right\} .
$$

Due to the uniqueness of the solution to (15) in $N^{\perp}$, the operator $Q(L u)$ is invertible in $N^{\perp}$. Then the solution $u(s)$ of (14) may be rewritten in the form

$$
u=U_{1}\left(U_{2}\right)+U_{2}
$$

where $U_{1}\left(U_{2}\right)$ is the solution of (18) for fixed but arbitrary $U_{2}$, and $U_{2}$ is afterwards obtained as a solution of (19) where $U_{1}=U_{1}\left(U_{2}\right)$ was substituted.

Now, we will compute $U_{1}, U_{2}$ solving problems (18), (19), or, more precisely, their approximations. Let us introduce a sufficiently small parameter $t$ and use the following series

$$
\begin{aligned}
\lambda-\lambda_{0} & ={ }_{1} t+{ }_{2} t^{2}+{ }_{3} t^{3}+\ldots, \\
u(s) & =v_{0}(s) t+u_{2}(s) t^{2}+u_{3}(s) t^{3}+\ldots, \\
\sin u & =u-\frac{u^{3}}{3 !}+\frac{u^{5}}{5 !}-\ldots
\end{aligned}
$$

in (12). Comparing the coefficients corresponding to the same power $t^{n}, n=1,2,3, \ldots$, we get an infinite system of equations, three of which are written below

$$
\begin{gathered}
v_{0}^{\prime \prime}(s)+\lambda_{0} s v_{0}(s)=0, \\
u_{2}^{\prime \prime}(s)+\lambda_{0} s u_{2}(s)=-{ }_{1} v_{0}(s), \\
u_{3}^{\prime \prime}(s)+\lambda_{0} s u_{3}(s)=-s\left(-\frac{\lambda_{0}}{2} v_{0}^{2}+{ }_{2} v_{0}+{ }_{1} u_{3}-\frac{1}{6} v_{0}^{3}\right) .
\end{gathered}
$$

Other equations may easily be obtained continuing the computations. (21) is automatically satisfied due to (11). Any solution of (21) is of the form (16) for $f(s)=-{ }_{1} v_{0}(s)$ satisfying (17), which implies ${ }_{1}=0$. In this case, the only solution $u_{2} \in \mathrm{N}^{\perp}$ is $\mathrm{u}_{2}(\mathrm{~s}) \equiv 0$. Substituting these values into (23), we get the equation for $u_{3}$ :

$$
u_{3}^{\prime \prime}(s)+\lambda_{0} s u_{3}(s)=s\left(\frac{\lambda_{0}}{2} v_{0}^{2}-{ }_{2} v_{0}\right), \quad s \in(0,1) .
$$

And again, any solution of (24) satisfying (8) is of the form (16) under the assumption (17) on the right hand side of (24), which determines the unique ${ }_{2}$. The constant $C$ from (16) is determined by the condition $u_{3} \in N^{\perp}$.

The computation of ${ }_{n}, u_{n}(s)(n=2,3, \ldots)$ gives approximations of the solution $u(s)$ of (14), whereas ${ }_{n}, u_{n+1}(s)$ is a zero for odd indices $n$. The approximations

$$
u(s) \doteq v_{0}(s) t+u_{3}(s) t^{3}
$$

for the values of parameter $t= \pm 0.5, \pm 1.0, \pm 1.5$ are given in Fig. 5.

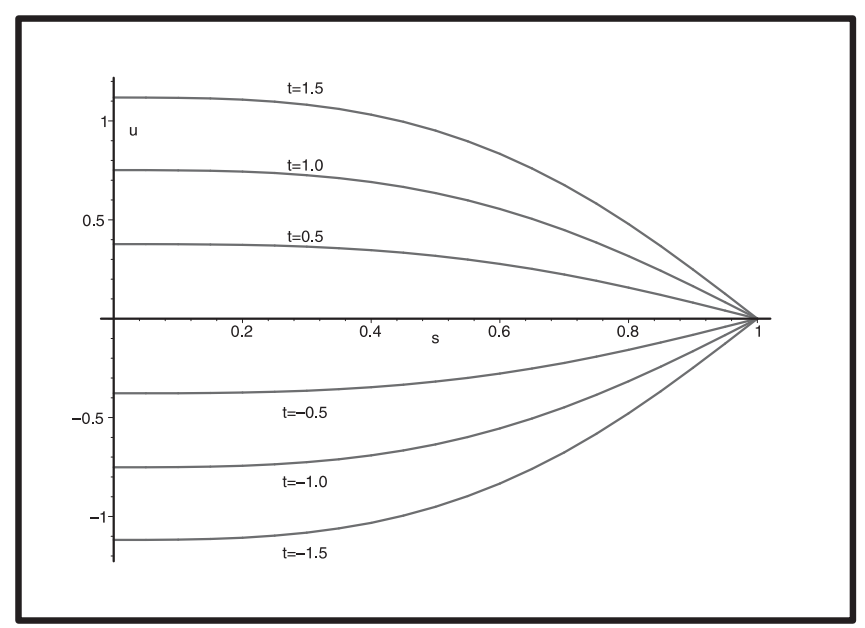

Fig. 5: The approximate solutions $u(s)$ for $t= \pm 0.5, \pm 1.0, \pm 1.5$

The dependence of two approximations of $\lambda$ (denoted by $L_{1}, L_{2}$ on the picture 6 ):

$$
\lambda=\lambda_{0}+{ }_{2} t^{2}, \lambda=\lambda_{0}+{ }_{2} t^{2}+{ }_{4} t^{4}
$$

is shown in Fig. 6.

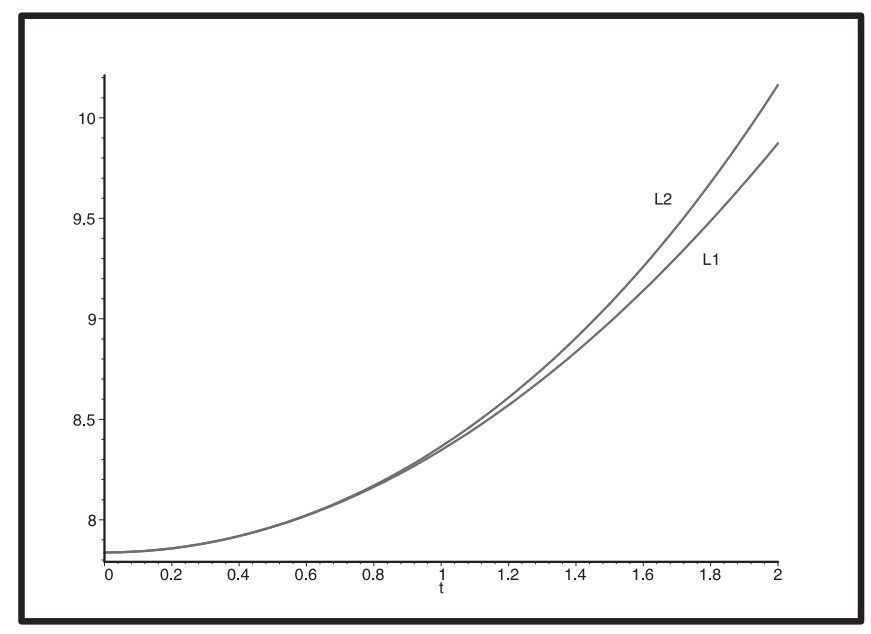

Fig. 6: Two approximations of $\lambda: L_{1}, L_{2}$

Excluding parameter $t$ from (25) and (26), we get the maximum angle $\varphi_{0}$ (denoted by $U_{0}$ in Fig. 7 ) as a function of the length $l$ of the column (see Fig. 7). 


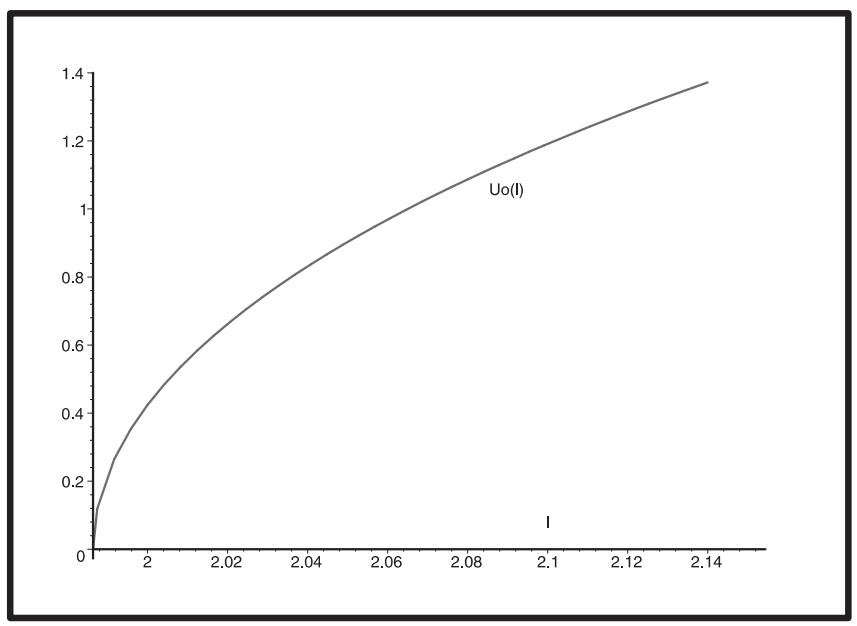

Fig. 7: Graph of the maximum angle $\varphi_{0}(l) \equiv \mathbf{u}(l)$

\section{Conclusions}

The last figure illustrates the fact that the nonlinear theory gives reasonable results:

a) the deflections of the column loaded with its own weight are zero for $\lambda \leq \lambda_{0}$ (the first eigenvalue of the linear problem),

b) the maximum deflection increases continuously with increasing length of the column.

Formula (25) represents the solution of the problem as the sum of the first eigenfunction $v_{0}(s)$ of the linear problem multiplied by parameter $t: t \rightarrow 0$ for $\lambda \rightarrow \lambda_{0}$ and "a perturbation", which is orthogonal to $v_{0}$ in $L^{2}$. The above mentioned method is applicable to other stability problems described by nonlinear ordinary and partial differential equations, e.g., lateral buckling.

\section{Acknowledgement}

The author is grateful to prof. J. Šejnoha for his stimulating discussions and valuable advice.

The research of the author was supported by the foundation of the Ministry of Education in the project "Výzkumný záměr No. J04/98:210000001”.

\section{References}

[1] Rzhanicyn, A. R.: Ustoichivost ravnoviesija uprugich sistem. Moskva 1955

[2] Collatz, L.: Eigenwertaufgaben mit technischen Anwendungen. Leipzig 1963

[3] NIRENBERG, L.: Topics in Nonlinear Functional Analysis. New York 1974

[4] Krasnoselskij, M. A., Zabrejko, P. P.: Geometricheskije metody nelinejnogo analiza. Moskva 1975

[5] Char, B. W., Geddes, K. O., Gonet, G. H., Leong, B. L., Monagan, M. B., Watt, S. M.: First Leaves: A Tutorial Introduction to Maple V. Springer-Verlag 1992

RNDr. Marie Kopáčková, CSc.

Department of mathematics phone:+420224354385

e-mail: marie.kopackova@fsv.cvut.cz

Czech Technical University in Prague

Faculty of Civil Engineering

Thákurova 7, 16629 Praha 6, Czech Republic 